\title{
Role of epigenetic factors in the selection of the alternative splicing isoforms of human KRAS in colorectal cancer cell lines
}

\author{
Ángela L. Riffo-Campos ${ }^{1,2}$, Francisco Gimeno-Valiente ${ }^{1}$, Fernanda M. Rodríguez ${ }^{1,3}$, \\ Andrés Cervantes ${ }^{1,4,5}$, Gerardo López-Rodas ${ }^{1,6}$, Luis Franco ${ }^{1,6}$ and Josefa Castillo ${ }^{1,5}$ \\ ${ }^{1}$ Institute of Health Research INCLIVA, Valencia, Spain \\ ${ }^{2}$ Present Address: Centro De Excelencia de Modelación Y Computación Científica, Universidad De La Frontera, Temuco, Chile \\ ${ }^{3}$ Instituto De Ciencias Veterinarias Del Litoral (ICIVET Litoral), Universidad Nacional Del Litoral (UNL)/Consejo Nacional De \\ Investigaciones Científicas Y Técnicas (CONICET), Santa Fe, Argentina \\ ${ }^{4}$ Department of Medicine, Universitat De València, Valencia, Spain \\ ${ }^{5}$ Centro De Investigación Biomédica En Red En Cáncer (CIBERONC), Madrid, Spain \\ ${ }^{6}$ Department of Biochemistry and Molecular Biology, Universitat De València, Valencia, Spain \\ Correspondence to: Luis Franco, email: Ivis.franco@uv.es \\ Keywords: epigenetics; chromatin structure; alternative splicing; colorectal cancer; KRAS isoforms \\ Received: November 03, $2017 \quad$ Accepted: March 17, $2018 \quad$ Published: April 17, 2018 \\ Copyright: Riffo-Campos et al. This is an open-access article distributed under the terms of the Creative Commons Attribution \\ License 3.0 (CC BY 3.0), which permits unrestricted use, distribution, and reproduction in any medium, provided the original author \\ and source are credited.
}

\section{ABSTRACT}

Mutation-driven activation of KRAS is crucial to cancer development. The human gene yields four mRNA splicing isoforms, 4A and 4B being translated to protein. Their different properties and oncogenic potential have been studied, but the mechanisms deciding the ratio 4A/4B are not known. To address this issue, the expression of the four KRAS isoforms was determined in 9 human colorectal cancer cell lines. HCT116 and SW48 were further selected because they present the highest difference in the ratio 4A/4B (twice as much in HCT116 than in SW48). Chromatin structure was analysed at the exon $4 \mathrm{~A}$, characteristic of isoform $4 \mathrm{~A}$, at its intronic borders and at the two flanking exons. The low nucleosome occupancy at exon 4A in both cell lines may result in a fast transcriptional rate, which would explain the general lower abundance of isoform $4 \mathrm{~A}$, also found in cells and tissues by other authors, but due to its similarity between both cell lines, chromatin structure does not influence alternative splicing. DNA methylation downstream exon 4A significantly differs in HCT116 and SW48 cells, but the CCCTC-binding factor, which affects the processivity of RNA polymerase and the alternative splicing, does not bind the differentially methylated sequences. Quantitative epigenetic analysis at mononucleosomal level revealed significant differences between both cell lines in H3K4me3, H3K27me3, H3K36me3, H3K9ac, H3K27ac and H4K20me1, and the inhibition of some histone-modifying enzymes alters the ratio 4A/4B. It can be concluded that the epigenetic modification of histones has an influence on the selection of isoforms $4 A$ and $4 B$.

\section{INTRODUCTION}

Human KRAS locus is located in chromosome 12 $(25,204,789-25,250,936)$ and is transcribed from the reverse strand. Four mRNA isoforms, which result from alternative splicing, are reported in the Ensembl Genome database (accession number ENSG00000133703). Two of these mRNA isoforms are translated to protein, giving rise to the well-known KRAS-4A and KRAS-4B products. The two remaining mRNA isoforms contain open reading frames and may be putatively translated, but the actual existence of their protein products has not been reported 
to date. The first 164 residues of KRAS-4A and KRAS-4B are identical, but the $\mathrm{C}$-terminal regions of the molecules (25 amino acids in isoform KRAS-4A and 24 in KRAS4B), encoded by different exons, show a high variability. KRAS-4A and KRAS-4B are members of the Ras protein family, which also includes the highly homologous HRAS and NRAS. All of these proteins display GTPase activity and are involved in signalling pathways that regulate many cellular processes, including cell proliferation.

The functional cellular environment of the KRAS isoforms is the plasma membrane $[1,2]$, in which they are anchored through the farnesyl chains posttranslationally added at a C-terminal motif [3]. Isoform $4 \mathrm{~A}$ is further directed to membrane by palmitoylation of a specific cysteinyl residue [4] and a sequence containing 7 lysines also contributes to the membrane localization of KRAS-4B through electrostatic interactions with the inner leaflet of the plasma membrane [2]. The function of the latter topogenic signal is regulated by the phosphorylation of serine 181, which is interspersed in the basic stretch [5]. KRAS-4A is directed to its final location via the Golgi system, while KRAS-4B goes from the endoplasmic reticulum to the plasma membrane through a different mechanism, which involves phosphodiesterase- $\delta[6,7]$. The structural bases for specific KRAS-4B/ phosphodiesterase- $\delta$ interaction have been recently studied [8].

The isoforms KRAS-4A and KRAS-4B behave in a different way in many other aspects. For instance, while the expression of $K R A S-4 B$ is ubiquitous in human tissues $[9,10]$, that of $K R A S-4 A$ is restricted to the gastrointestinal tract, kidney, lung and other tissues of endodermal origin [9] and similar results were obtained in mice [11, 12]. As to the oncogenic potential of both isoforms there is some discrepancy in the data recorded in the literature. It is clear that the ratio of both isoforms is altered in cancer [9] and, while the focus-inducing potential of KRAS-4B is lower than that of KRAS-4A, the latter isoform do not induce cell migration and KRAS-4B does [13]. These early results are in agreement with those of King et al., who found that knockdown of KRAS-4A reduces proliferation of renal cell carcinoma [10]. However, after the results obtained in mice, KRAS-4A is classically considered to be proapototic, whereas KRAS-4B is antiapoptotic [14].

At any rate, it is clear that changes in the ratio of splicing isoforms $4 \mathrm{~A}$ and $4 \mathrm{~B}$ exist when comparing their expression levels in different cell lines or tissues, but the results reported in the literature are also controversial. It has been described that the ratio $K R A S-4 A / K R A S-4 B$ is significantly reduced in $6 \mathrm{CRC}$ cell lines when compared with cells derived from normal colon and in tumour from 4/9 patients with sporadic CRC when compared with the adjacent normal mucosa [9] and similar results were obtained in adenomas of mouse small intestine in the absence of $K R A S$-activating mutations [15]. In agreement with these results, Chung et al., by transfecting mouse livers with different $K R A S$ constructs, found that the survival of mice carrying mutation-activated $K R A S-4 A$ is significantly better than that of animals transfected with mutated $K R A S-4 B$ [16] and Luo et al. propose that the exon specifically included in $K R A S-4 A$ confers the gene a certain tumour-suppressor function [17]. The above data suggest that mutation-activated $K R A S 4 B$ is more oncogenic than activated $K R A S 4 A$, and yet there are some discordant views in the literature. For instance, Wang et al. reported that increase of the ratio $K R A S-4 A / K R A S-4 B$ in murine lung correlated with higher susceptibility to tumour development [12] and a major role in mouse lung carcinogenesis has been ascribed to $K R A S-4 A$ [18]. It has also been described that the expression of this isoform in human colorectal cancer and adenomas is higher than in the adjacent normal tissues [19].

Whatever the causes of the above discrepancies, it seems clear that it is worth studying the factors that decide the proportion of $K R A S$ isoforms. Differential splicing is affected by many factors, including chromatin structure and epigenetic modifications near the splicing sites (for reviews, see $[20,21])$. None of these factors have been studied in the human $K R A S$ locus, although there is evidence that cis-acting elements, presumably located in introns or in 3'-untranslated regions decide the balance between $K R A S-4 A$ and $K R A S-4 B$ in murine lung cancer [22]. These circumstances prompted us to investigate the distribution of $K R A S$ isoforms, including the non-coding ones, which might possess a regulatory role, in human CRC cell lines and to determine the chromatin structure and its epigenetic modifications in the regions involved in the selection among the different isoforms.

\section{RESULTS}

\section{Expression of $K R A S$ isoforms in different cell lines}

To facilitate the interpretation of the results, a map of the human KRAS locus is given in Figure 1. The four possible transcripts are identified by the isoform number and code included in the ENSEMBL database. The conventional exon numbering, which correspond only to the translated ones (see, for instance, ref. [23]), is used. The untranslated exon present upstream of exon 1 in all the isoforms is further referred to as exon 0 and the exon downstream exon 1 , characteristic of isoform 2 , is designed as $1^{\prime}$.

We first quantified the transcription of whole $K R A S$ in $9 \mathrm{CRC}$ cell lines (Figure 2A) using primers from exon 0 , common to all mRNA isoforms. The relative abundance of each four transcripts was then determined (Figure 2B). To differentiate among the four isoforms we designed the primers given in Supplementary Table 1. Isoform 1 (4B) was analysed with a forward primer from exon 3 and a reverse primer spanning exons 3 and 4B. Isoform 2 was quantified by using a forward primer from exon 
1 and a reverse primer from exon 1', which is unique to this isoform. To analyse isoform 3, a forward primer from exon 1 and a reverse one spanning exons 1 and 4B were used. Finally, isoform 4 (4A) was quantified with a forward primer from exon $4 \mathrm{~A}$, which is present only in this isoform, and a reverse primer from 5 ' end of exon $4 \mathrm{~B}$. The location of those primers is depicted in Supplementary Figure 1. To correct for the possible differences in the efficiency of the primers, we determined the efficiency factors, which are also given in Supplementary Table 1. The slight differences in efficiency did not affect the results of the RT-qPCR experiments given in Figure 2B.

The expression of whole $K R A S$ is highly variable from line to line, being higher in Caco 2 and lower in RKO and SW48. The expression level is roughly similar in lines HCT116 and DLD1 and the cells derived from these parental lines show a lower expression level, although a slight compensatory effect for the loss of one of the alleles seems to occur (Figure 2A). The expression of the individual isoforms, relative to $A C T B$, is given in Figure 2B. Isoform 1 (4B) is the most abundant in all the cell lines studied; present results agree with those of Tsai et al. [23]. The level of isoform 3 is negligible in all the cell lines, but the expression of the non-translated isoform 2 is comparable, or even slightly higher than that of isoform 4 (4A).

In view of the above mentioned interest of studying the mechanisms deciding the relative abundance of the different splicing isoforms of $K R A S$, especially the isoforms 1 (4B) and 4 (4A), we selected the cell lines SW48 and HCT116. This selection was based in the fact that they are the lines showing a greater difference in the ratio between both isoforms. This is especially apparent in Figure $2 \mathrm{C}$, in which the percentage of the four isoforms is given. The percentage of isoform 4 (4A) in HCT116 doubles that in SW48 at the expense of isoform 1 (4B),

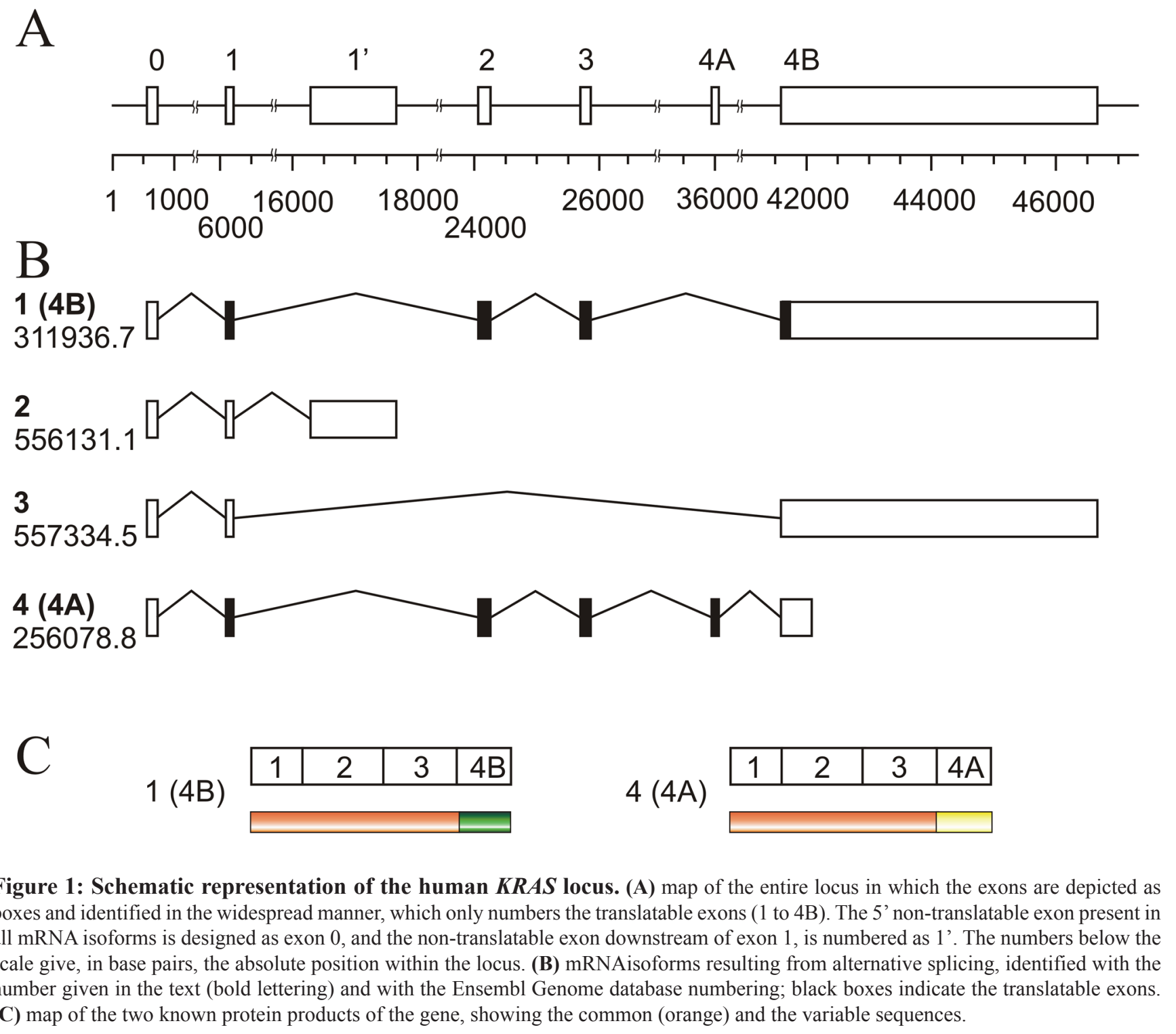




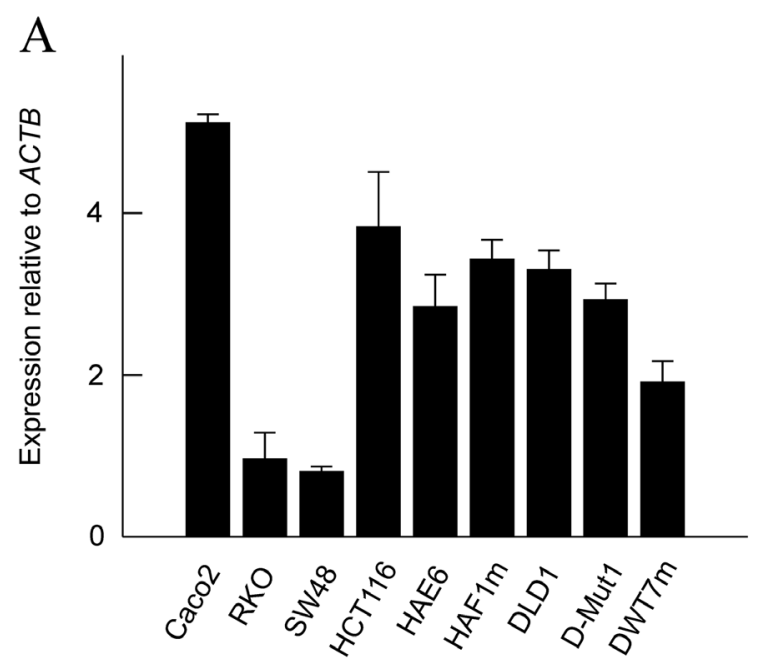

B

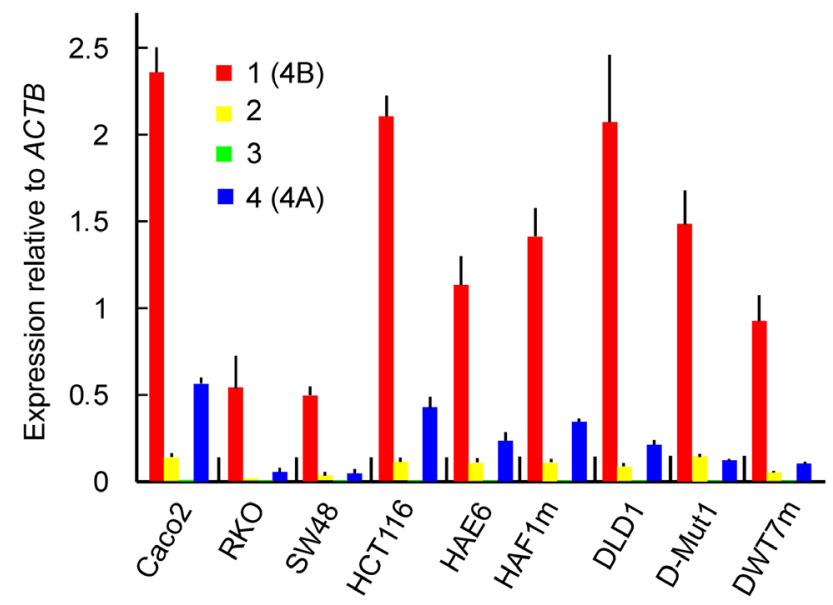

$\mathrm{C}$

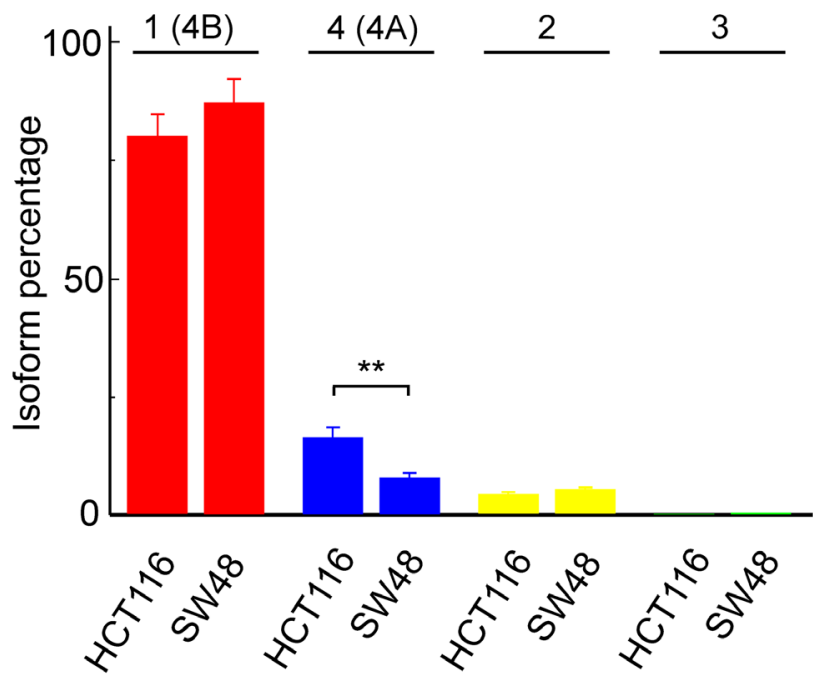

Figure 2: Transcription level of the whole $K R A S$ gene and of its isoforms in several human CRC cell lines. (A) expression, relative to the $A C T B$ gene, of the whole gene in 9 cell lines. (B) expression, relative to the $A C T B$ gene, of the four isoforms in those cell lines. (C) percent expression of the four isoforms in HCT116 and SW48 cells. The results were obtained by RT-qPCR in triplicate and expressed as the mean \pm standard deviation. 
while the proportion of isoform 2 is roughly similar in both cell lines and that of isoform 3 is negligible. In other words, exon 6 (4A) is skipped in SW48 cells twice more than in HCT116 cells.

\section{Chromatin structure at the differential splicing sites of HCT116 and SW48 cell lines}

Taking into account that the chromatin structure is a factor influencing the splicing events [20], the nucleosomal organization of the KRAS locus in exons 3 and $4 \mathrm{~A}$ and in the translatable $5^{\prime}$ end of exon $4 \mathrm{~B}$ was studied. These regions include the adjacent splicing sites and other potential sequences that may influence inclusion or skipping of exon 4A. The micrococcal nuclease protection data, obtained at the amplicons defined in Supplementary Table 2, are compatible with the occupancy of the three exons by nucleosomes. The size of the exons is, respectively, 165, 124 and $117 \mathrm{bp}$, so there is room in them for a single nucleosome, and the width of the protected areas are also compatible with the presence of a single nucleosome over each exon (Figure 3A-3C). The sequence-based prediction retrieved from the NuPop program is compatible with the presence of nucleosomes over exons 3 and $4 \mathrm{~A}$, but not with the presence of a nucleosome in the 5' end of exon 4B, in which the probability of assembling a nucleosome is very low, in accordance with the high content of $\mathrm{A}$ and $\mathrm{T}$ in that region. In spite of these circumstances, the results suggest that the nuclease protection observed in the 5 ' end of exon 4B (Figure 3C) is actually due to the presence of a nucleosome. As it will be shown later, the Nuc-ChIP experiments gave positive results at the amplicon centred at position 41600 and a quantitative determination of the concentration of H3 by using a Nuc-ChIP assay with an antibody against the C-terminal end of the histone gave at least twice as much $\mathrm{H} 3$ over the exon $4 \mathrm{~B}$ than over the exon 4A. Thus, it is highly probable that the nuclease protection at the 5 ' end of exon $4 \mathrm{~B}$ is due to the presence of a bona fide nucleosome.

The profiles of micrococcal nuclease protection are almost identical in HCT116 and SW48 cell lines, and only a slight difference is observed in the nucleosome occupancy over exon 4B. Within the resolution margin of this assay, the flanks of the exons, which contain the 3' and 5' splicing sites, as well as the polypyrimidine tract immediately upstream of the 5' sites, seem to be unprotected in both cell lines and, then, accessible to the spliceosome assembly. In view of the above results, it seems that the chromatin structure has no influence on the differences in exon 4A skipping between both cell lines.

\section{DNA methylation downstream of exon $4 \mathrm{~A}$}

DNA methylation can also affect exon skipping in several ways [24]. To check whether distinct methylation levels may be the cause of the differential level of $K R A S$ isoforms 4 (4A) and 1 (4B) in HCT116 and SW48, we analysed DNA methylation downstream of exon 4A. As shown in Figure 4, there is a single $\mathrm{CpG}$ dinucleotide within exon $4 \mathrm{~A}$ and this low $\mathrm{CpG}$ content may be related to its low nucleosome occupancy (see Figure 3B) [25]. Several $\mathrm{CpG}$ sites are clustered in the downstream intron starting approximately at $500 \mathrm{bp} \mathrm{3'}$ to exon 4A, but only 6 out of these sites could be analysed to quantify their methylation degree following the used procedure (Figure 4B). Subtle, but significant, differences between SW48 and HCT116 cell lines were observed in the three CpG sites analysed between 36700 and 36870, which showed a more intense methylation in HCT116 (Figure 4A).

The possibility that these differences in methylation resulted in a distinct CCCTC-binding factor (CTCF) recruitment was next studied, as it has been described that the presence of bound CTCF downstream of exons
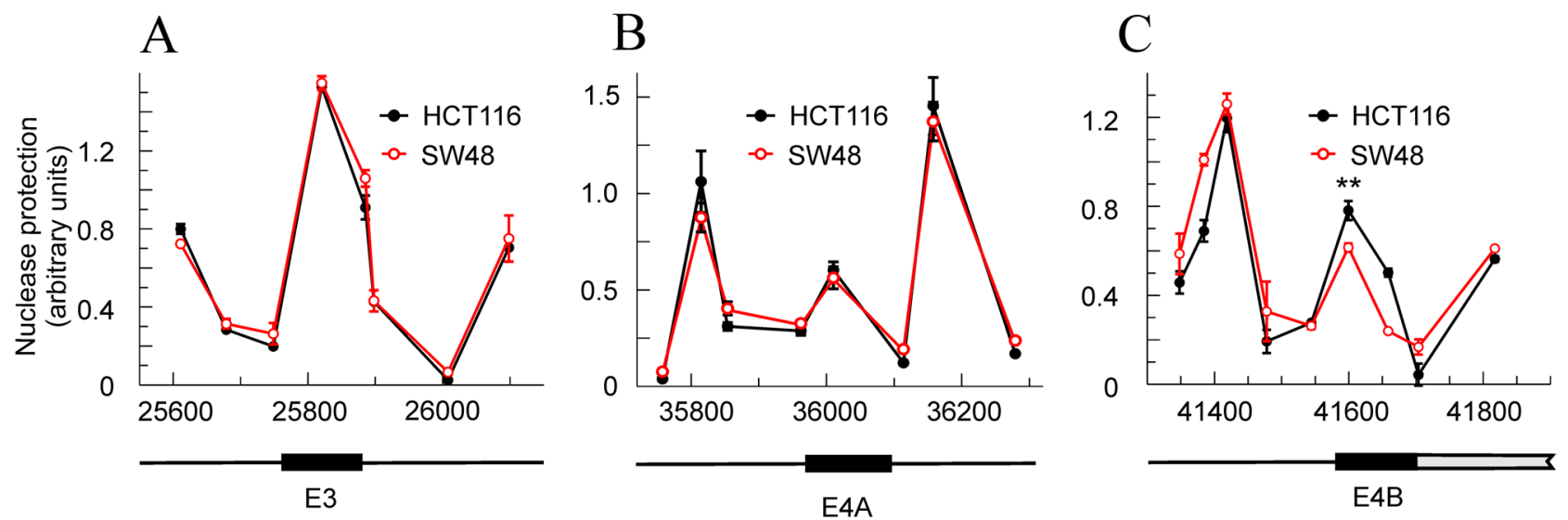

Figure 3: Nucleosome occupancy at the exons involved in alternative splicing in HCT116 and SW48 cells. (A) exon 3 . (B) exon 4A. (C) exon 4B. The plots give the nuclease protection in arbitrary units against the position of each amplicon centre in the locus. The maps below identify the exon location as black rectangles. In (C), the gray rectangle refers to the non-translatable, transcribed exon. The results correspond to three determinations for each amplicon (Panels A and C) or to six determinations from two independent experiments (Panel B) and are given as the mean \pm standard deviation. ${ }^{* *} \mathrm{p}<0.01$. 
causes a diminution of the elongation rate of RNA pol II, which in turn may affect the inclusion of the exon due to kinetic factors [24]. ChIP analysis, followed by qPCR evaluation of amplified sequences, revealed that CTCF does not appreciably bind the region studied. Actually, the concentration of DNA sequences immunoprecipitated with an anti-CTCF antibody is similar to that recovered without added antibody (Supplementary Table 3).

\section{Epigenetic modification of histones in the regions involved in alternative splicing}

Taking into account that splicing usually is a cotranscriptional event, it is known that the presence of some epigenetic marks in nucleosomes surrounding the splicing sites may influence exon skipping and/or inclusion [20, 21]. As the differences in the selection between the $4 \mathrm{~A}$ and 4B KRAS isoforms in HCT116 and SW48 cell lines is not probably determined by chromatin structure or CTCF binding, the differential presence of epigenetic marks in the nucleosomes covering exons 3,4A and 4B was next studied by the Nuc-ChIP procedure. The following histone post-translational modifications (PTMs) were analysed:
H3K4me3, H3K9me3, H3K27me3, H3K36me3, H3K9ac, $\mathrm{H} 3 \mathrm{~K} 27 \mathrm{ac}$ and H4K20me1. These histone PTMs rank among the marks most frequently associated with splicing events (see [26] and references therein). Two independent Nuc-ChIP experiments were carried out at the amplicons corresponding to the three nucleosomes positioned on exons 3, 4A and 4B. The PCR quantifications were done in triplicate in both experiments. With only an exception (see below), the differences in the studied epigenetic marks between HCT116 and SW48 cells were not significant in exons 3 and $4 \mathrm{~A}$, while most of them were significant in the nucleosome covering exon 4B.

Only the results giving significant differences between HCT116 and SW48 cell lines are depicted in Figure 5. The comparison of the results with the marks on $\mathrm{H} 3 \mathrm{~K} 27$ provides an internal control of the validity of the experiments, because the trends of acetylation and methylation run in the opposite direction, as expected for two mutually exclusive PTMs.

$\mathrm{H} 3 \mathrm{~K} 27 \mathrm{ac}$ is the only epigenetic mark which shows significant differences in the three nucleosomes studied, being more intense in the three exons in HCT116

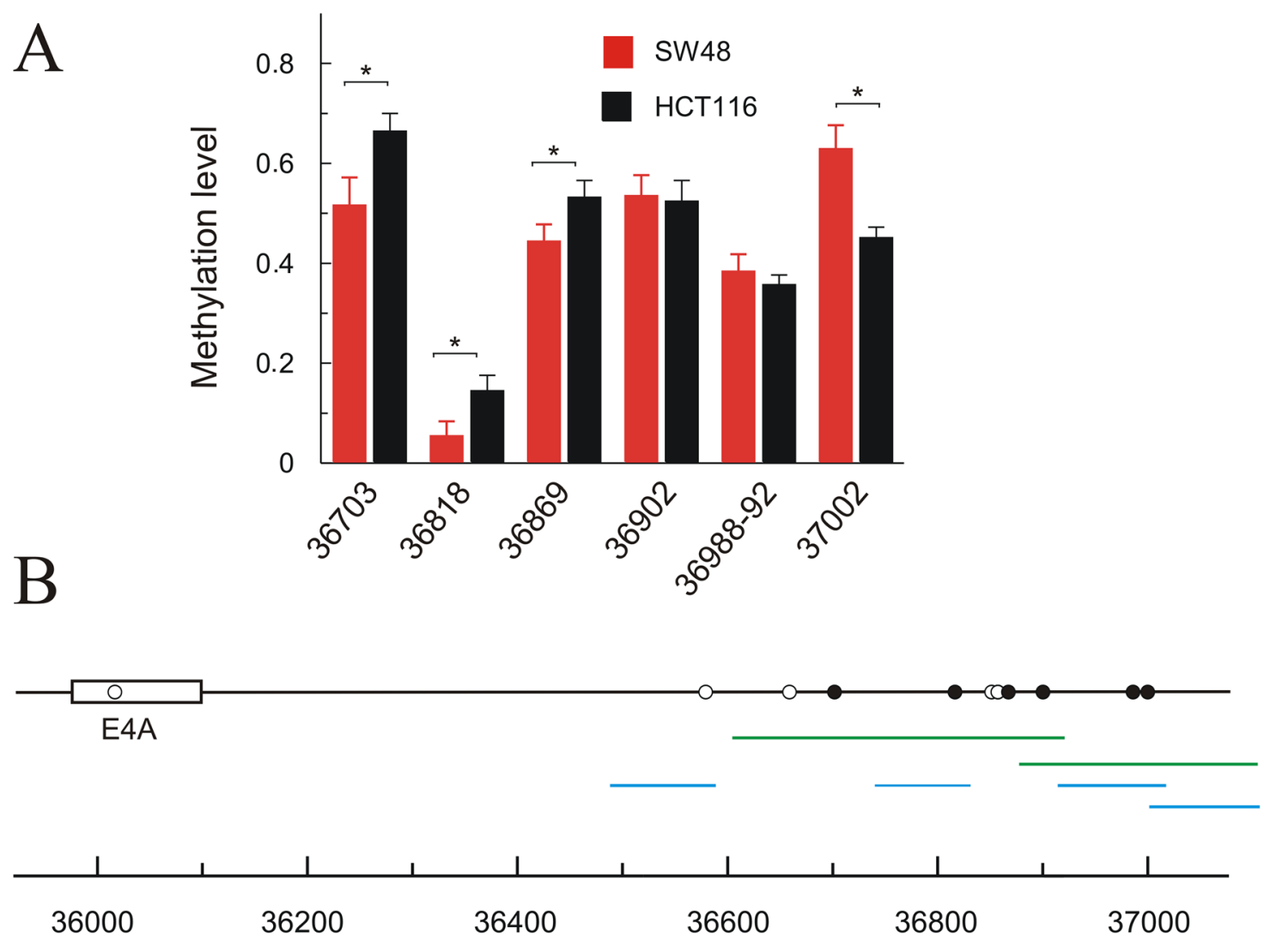

Figure 4: Methylation level of CpGs downstream exon 6 (4A) in HCT116 and SW48 cell lines. (A) the level of methylation in a quantitative scale (average of 5-10 determinations) is plotted against the position of the analysed CpGs in the locus; the CpGs showing a significant difference $(\mathrm{p}<0.05)$ between both cell lines are identified. $(\mathbf{B})$ map showing the position of the CpGs; filled circles indicate those analysed for their methylation level, while empty circles refer to those CpGs, which could not be analysed by the method used. The amplicons used to evaluate $\mathrm{CpG}$ methylation are shown in green, and those used for qPCR analysis of CTCF binding are represented in blue. 
cells. These cells are also more heavily marked in the nucleosome covering exon $4 \mathrm{~B}$ in four out of the other histone PTMs that show significant differences, namely H3K4me3, H3K36me3, H3K9ac and H4K20me1. Only $\mathrm{H} 3 \mathrm{~K} 27 \mathrm{me} 3$ is more intense in SW48 than in HCT116 (Figure 5). To check whether differences in histone epigenetic modifications are actually related to alternative splicing, the ratio of isoforms $4 \mathrm{~A} / 4 \mathrm{~B}$ was studied after inhibiting histone deacetylases with trichostatin A (TSA), and EZH1/2 histone methyltransferases with EPZ005687. Inhibition of the latter enzymes was selected because they specifically catalyse the methylation of $\mathrm{H} 3 \mathrm{~K} 27$. As the other methylatable lysines studied in the experiment of Figure 5 are modified by several redundant methyltransferases [27], inhibition of all of them results in cell lethality. As expected, treatment with TSA increases the acetylation level of bulk H3 and EPZ005687 reduces the methylation of H3K27 (Supplementary Figure 2). Figure 6 shows that reducing the level of H3K27me3 causes a significant increase in the $4 \mathrm{~A} / 4 \mathrm{~B}$ ratio in the SW48 cell line. In both cell lines, the TSA-induced change of $\mathrm{H} 3$ acetylation leads to significant changes in the $4 \mathrm{~A} / 4 \mathrm{~B}$ ratio, which resulted levelled after the treatment.

\section{DISCUSSION}

The results described in the present paper add some details to our knowledge on the distribution of $K R A S$ isoforms and on the mechanisms involved in their selection. First, in spite of the differences in the expression of the whole gene observed among the different cell lines analysed (Figure 2A), isoform 1 (4B) is the more abundant in every case (Figure 2B). With a few exceptions, this is a common feature in both, cell lines and human normal and cancerous tissues $[9,17,23]$. The splicing variants 2 and 3 have received little or no attention in the literature. The fact that the translation of their mRNAs had not been demonstrated to date may be a cause for that lack of interest.

As far as we know, present paper describes for the first time a study of the distribution of those isoforms in several CRC cell lines. The presence of isoform 3 is negligible in all the cell lines studied, but isoform 2, which includes exon 1 ' and a premature transcription termination signal (Figure 1), is clearly detectable. It is known that a non-translatable mRNA splice variant of $H R A S$, which includes the otherwise skipped exon 5, plays a regulatory role in the expression of the gene [28], and it would be interesting to know whether $K R A S$ isoform 2 performs a similar role.

The differences in the ratio between isoforms 1 (4B) and 4 (4A) among the different cell lines found in the present paper, as well as the differences observed by many authors, put forward the question as to what splicing regulatory mechanisms decide between the inclusion or skipping of exon $4 \mathrm{~A}$ in the mature transcript. In fact, exons $4 \mathrm{~A}$ and $4 \mathrm{~B}$ cannot be regarded as mutually exclusive exons at the level of mature mRNA, because the 5' end of exon $4 \mathrm{~B}$ is present in the mature transcript of both isoforms 1 (4B) and 4 (4A). In the latter isoform, exon 4B is not translated, because the included exon $4 \mathrm{~A}$ contains a stop codon (TAA) in the 3 ' end.

Intron and exon definition may obey to two different mechanisms, occurring either at the level of the mRNA precursor, or at the DNA level [21]. Taking into account that in most cases splicing is a cotranscriptional event, the second mechanism seems to be the most common one. In this case, chromatin structure plays a fundamental role. Nucleosomes are usually positioned in exons and their presence causes RNA polymerase to reduce its processivity [29], helping the splicing factors to be recruited to the nascent pre-mRNA [21].

To investigate the mechanisms deciding the inclusion of exon 4A, a comparison of the chromatin

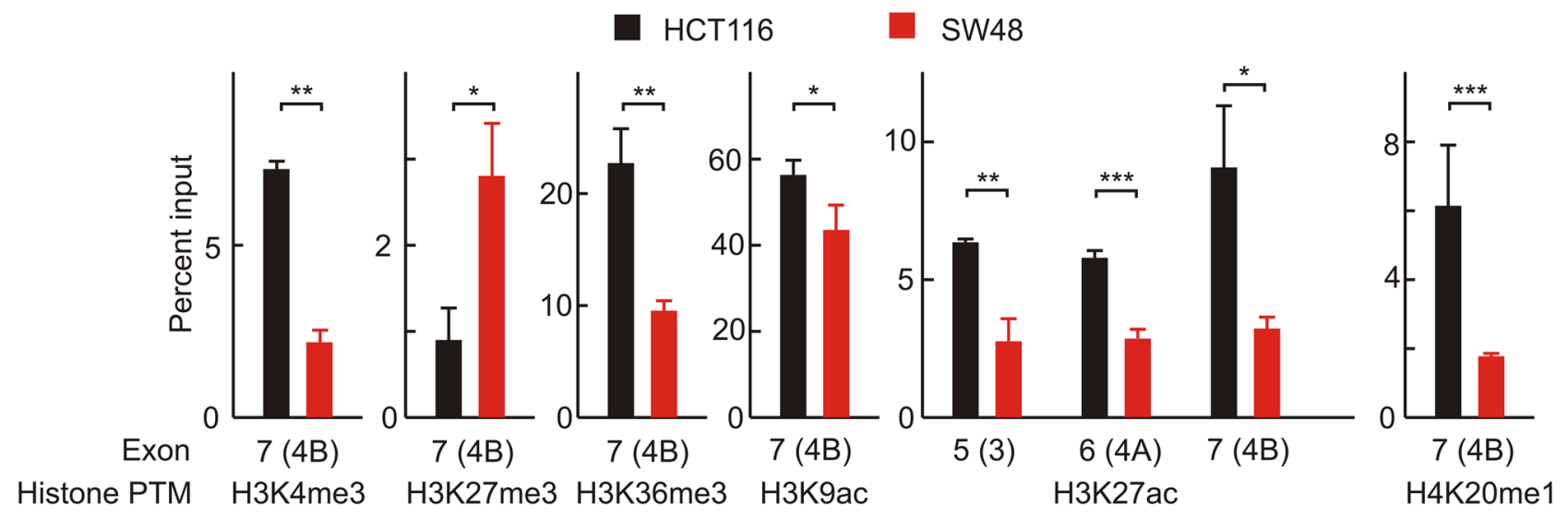

Figure 5: Nuc-ChIP analysis of histone epigenetic modifications in nucleosomes located over the exons 3 , $4 \mathrm{~A}$ and $4 \mathrm{~B}$. Only those histone PTMs that significantly differ between HCT116 and SW48 cells are depicted. Three independent Nuc-ChIP experiments were carried out and the figure gives the results of one representative experiment. qPCR analysis were carried in triplicate and the mean \pm standard deviation is plotted. ${ }^{*} \mathrm{p}<0.05 ;^{* *} \mathrm{p}<0.01$; $^{* * *} \mathrm{p}<0.001$. 
structure of the HCT116 and SW48 cells was first done. Of note, although the differences between both cell lines are negligible, the nucleosome occupancy is lower at exon $4 \mathrm{~A}$ than at the other exons. It is also much lower than in the proximal regions of the flanking introns (Figure 3B), so the processivity of RNA polymerase II is not reduced at its passage through exon 4A. Interestingly, the presumably fast processivity of RNA polymerase II through exon 4A may explain why the isoform containing this exon is less abundant than isoform 1 (4B) in all the cell lines studied in the present paper as well as in the patient samples and cell lines analysed by other authors [23].

The epigenetic differences between HCT116 and SW48 cell lines in the regions of interest to the present study were also examined. It is known that CpG methylation of DNA and histone PTMs can affect splicing through the "recruitment coupling" model [20]. For instance, DNA methylation at $\mathrm{CpG}$ dinucleotides prevents the binding of $\mathrm{CTCF}$ at overlapping target sites of the factor. As CTCF represents an obstacle to RNA polymerase II processivity, CTCF binding at nonmethylated $\mathrm{CpG}$ containing sites favours the inclusion of weak upstream exons [30]. Nevertheless, the present results (Figure 4 and Supplementary Table 3) allowed us to conclude that CTCF-dependent kinetic factors do not represent a main mechanism to decide the inclusion of exon $4 \mathrm{~A}$.

Finally, the histone PTMs were analysed by Nuc$\mathrm{ChIP}$ in the three nucleosomes positioned over the exons $3,4 \mathrm{~A}$ and $4 \mathrm{~B}$. We have previously used this technique $[31,32]$ and its advantages have been recently reviewed
[33]. Most of the significant differences in histone PTMs between HCT116 and SW48 cell lines are found in the nucleosome positioned in exon 4B (Figure 5).

The experiment of Figure 6 supports the hypothesis that changes in histone PTMs result in an alteration of the alternative splicing events. The enhancement of the acetylation of $\mathrm{H} 3$ is associated with a significant increase in the 4A/4B ratio in SW48 cell line (Figure 6B), in which the low level of $\mathrm{H} 3$ acetylation (Figure 5) is linked to a low $4 \mathrm{~A} / 4 \mathrm{~B}$ ratio. SW48 cells are characterized by a high level of $\mathrm{H} 3 \mathrm{~K} 27 \mathrm{me} 3$ (Figure 5) and a lower 4A/4B ratio and, therefore, a diminution of methylation might well be linked to an increase of the isoform ratio. The effects of inhibiting EZH1/2 histone methyltransferases on HCT116 cells are not so easy to explain. Anyway, the results of Figure 6 clearly shows that the histone PTMs examined are in some way responsible for the discrimination between KRAS 4A and 4B isoforms.

It is not an easy task to ascribe the differences in the isoform ratio to a given epigenetic mark. In spite of the efforts that have been carried out in the last years to find a splicing code (for an early proposal, see [34]), an unambiguous correlation between histone epigenetic marks and splicing events does not exist. Many of the studies in that sense have been carried out by means of genomic approaches [35] and, while the information provided by these methods is highly valuable from a statistical point of view, particular genes may not obey to those general rules.

The differences in histone PTMs between HCT116 and SW48 cells shown in Figure 5 are mainly confined
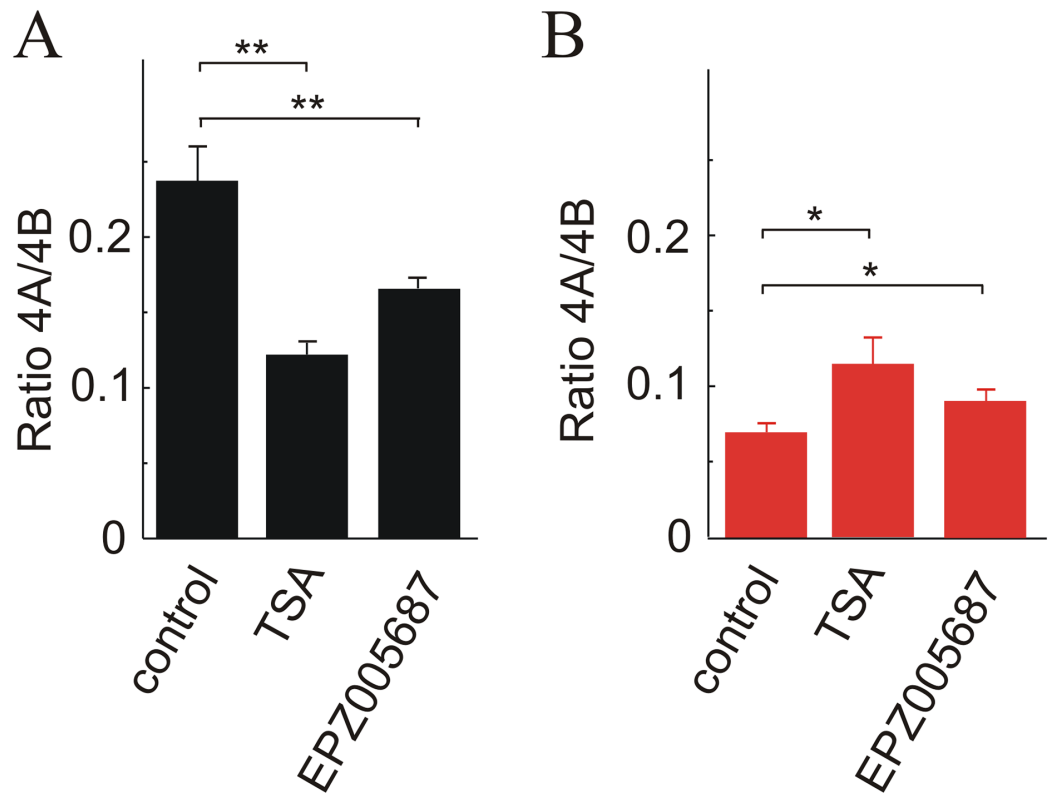

Figure 6: Effects of the changes in the level of $\mathrm{H3}$ acetylation and $\mathrm{H} 3 \mathrm{~K} 27$ methylation on the $K R A S$ 4A/4B ratio. TSA was used to inhibit histone deacetylases and EPZ005687 to inhibit EZH histone methyltransferases. (A) HCT116 cell line. (B) SW48 cell line. 
Table 1: Cell lines used in this paper

\begin{tabular}{|c|c|c|c|c|c|}
\hline \multirow{2}{*}{ Cell line } & \multicolumn{4}{|c|}{ Relevant genotype } & \multirow{2}{*}{ Source } \\
\hline & $K R A S$ & $B R A F$ & PIK3CA & TP53 & \\
\hline $\begin{array}{l}\text { DLD1 } \\
\text { D-Mut1 } \\
\text { DWT7m }\end{array}$ & $\begin{array}{c}\text { G13D/wt } \\
\text { G13D/- } \\
\text { G12D*- }\end{array}$ & wt & E545K; D549N & $\mathrm{S} 241 \mathrm{~F}$ & $\begin{array}{l}\text { ATCC CCL-221 } \\
\text { Dr Vogelstein } \\
\text { Riffo-Campos et } \\
\text { al. }[24]\end{array}$ \\
\hline $\begin{array}{l}\text { HCT116 } \\
\text { HAE6 } \\
\text { HAF } 1 \mathrm{~m}\end{array}$ & $\begin{array}{c}\text { G13D/wt } \\
\text { G13D/- } \\
\text { A146T/- }\end{array}$ & wt & H1047R & wt & $\begin{array}{l}\text { ATCC CCL-247 } \\
\text { Johns Hopkins } \\
\text { University } \\
\text { Roda et al. [23] }\end{array}$ \\
\hline RKO & wt & V600E & H1047R & wt & Horizon Discovery \\
\hline SW48 & wt & wt & wt & wt & Horizon Discovery \\
\hline Caco2 & wt & wt & wt & E204X & ATCC HTB-37 \\
\hline
\end{tabular}

The parental cell lines are represented in bold characters and, when appropriate, the mutant variant is listed below them. The mutation marked by an asterisk affects only $20 \%$ of cell population.

to the nucleosome over the exon 4B. The question arouses as to whether epigenetic differences in exon 4B may cause, in some way, the observed differential skipping of exon 4A. The recruitment model [36-38] provides a plausible hypothesis to explain the influence of epigenetic modifications of the nucleosome over exon $4 \mathrm{~B}$ on the skipping of the upstream exon. This model assumes that some histone PTMs recruit, through an adaptor, a splicing regulator that, in turn, binds a target regulatory site in nascent pre-mRNA. The present fine analyses at mononucleosomal resolution may be relevant to deepen the knowledge of the splicing epigenetic code and of the relationships between histone modifications and cancer progression. In this way, novel candidate therapeutic targets might be eventually found, by using either epigenetic modulators [39], or drugs targeted to the splicing machinery [40].

\section{MATERIALS AND METHODS}

\section{Cell lines}

The human CRC cell line HCT116 (ATCC CCL247) and their derivatives HAE6 (a gift from Dr B. Vogelstein) and HAF1m [41], DLD1 (ATCC CCL221) and their derivatives D-Mut1 (a gift from Dr B. Vogelstein) and DWT7m [42] were grown in McCoy's 5A medium (Sigma, St. Louis, MO, USA). The cell lines RKO (Horizon Discovery, Cambridge, UK) and Caco-2 (ATCC HTB-37) were grown in Dulbecco's modified Eagle's medium (DMEM) and the cell line SW48 (Horizon), was grown in RPMI 1640 medium (Sigma). All media were supplemented with $10 \%$ heat inactivated foetal bovine serum, $1 \%$ penicillin-streptomycin and 1\% L-glutamine (Sigma), and the cultures were maintained under standard conditions. Table 1 shows the relevant genotypes and sources of the cell lines. The cell lines obtained from other authors were not further authenticated.

To inhibit histone modifying enzymes, cells were loaded in 6-well plates and treated with either $0.25 \mu \mathrm{M}$ TSA (Sigma) or $20 \mu \mathrm{M}$ EPZ005678 (Selleckchem) for $24 \mathrm{~h}$.

\section{Determination of transcript levels}

RNA extraction, retrotranscription to cDNA and qPCR were carried out as previously described [42], using the $\beta$-actin gene $(A C T B)$ as standard. The primers used for whole $K R A S$ and for the different transcripts resulting from alternative splicing are given in Supplementary Table 1. The isoforms are identified by their number in the ENSEMBL database, followed in the case of isoforms 1 and 4 , by the more widespread nomenclature $4 \mathrm{~B}$ and $4 \mathrm{~A}$ in brackets.

To determine the efficiency of each qPCR primer set, a standard curve was prepared. The $\mathrm{Ct}$ values for serial template dilutions of cDNA are plotted against the logarithm of the dilution factor and the calculated slope gives a measure of the efficiency factor, which was used for normalization to compare the results obtained at different amplicons.

\section{Analysis of chromatin structure}

Nucleosome occupancy was determined by the micrococcal nuclease protection assay [32], using tiled amplicons of about $100 \mathrm{bp}$ in size. The primers used are given in Supplementary Table 2. The experimental results were compared with the output of the sequence-based prediction of positioning carried out using the NuPoP software tool [43]. 


\section{Analysis of DNA methylation}

Quantitative DNA methylation analysis was carried out essentially as described by Coolen et al.[44]. Briefly, the method uses a T7-promoter-tagged PCR amplification of bisulphite-modified DNA, followed by generation of a single-stranded RNA molecule and subsequent base-specific cleavage ( 3 ' to either rUTP or rCTP) by RNase A. The mixture of cleavage products, which differ in length and mass, are analysed by MALDI-TOF-MS. Changes in nucleotide sequence after bisulphite treatment, which reflects the differences in the methylation profile of original DNA, give origin to different fragment masses in the assay. The abundance of each fragment (signal/noise level in the spectrum) is indicative of the amount of DNA methylation in the analysed sequence.

Previously, bisulphite treatment of genomic DNA $(1 \mu \mathrm{g})$ was carried out with the EZ-96 DNA methylation kit (Zymo Research), following the manufacturer's protocol. For quantitative methylation analysis an AGENA's MassARRAY platform was used. PCR primers for the amplification of the different regions of the KRAS locus downstream of exon $4 \mathrm{~A}$ were designed by using Epidesigner (AGENA) and their sequences and location were given, respectively, in Supplementary Table 4 and Figure 4B.

The PCRs were carried out in a $5 \mu$ format with $10 \mathrm{ng} / \mu \mathrm{l}$ bisulfite-treated DNA, 0.2 units of TaqDNA polymerase (AGENA), $1 \times$ Taq buffer, and $200 \mathrm{nM} \mathrm{PCR}$ primers. Dephosphorylation of unincorporated dNTPs was performed as recommended by the manufacturer. The reaction mixtures were further diluted with $20 \mu \mathrm{l}$ of $\mathrm{H}_{2} \mathrm{O}$ and conditioned with $6 \mathrm{mg}$ of CLEAN Resin (AGENA) for optimal mass-spectra analysis.

\section{Chromatin immunoprecipitation (ChIP)}

To investigate the binding of CCCTC-binding factor (CTCF) to chromatin, ChIP analysis was carried out after sonicating the chromatin to an average fragment size of 250-300 bp following the previously described procedure $[45,46]$. The sequences of primers used for qPCR were given in Supplementary Table 5 and their location is depicted in Figure 4B. Although the amplicons are not tiled, the average size of chromatin fragments ensured that $\mathrm{CTCF}$ is absent from the entire region under consideration. Epigenetic modifications of histones were studied at nucleosomal level by Nuc-ChIP [31, 32]. The following antibodies were used: anti-H3K9ac (Abcam, ab-4441); anti-H3K9me3 (Abcam, ab-8898); anti-H3K27ac (Abcam, ab-4729); anti-H3K27me3 (Millipore, 07-449); anti-H3K4me3 (Abcam, ab-8580); anti-H3K36me3 (Abcam, ab-9050); anti-H3K20m (Abcam, ab-9051); anti- $\beta$-actin (Abcam, ab-8227).

\section{Statistical analysis}

Quantitative values are expressed as mean $\pm \mathrm{SD}$. Data in the different PCR determinations were compared by two-tailed t-test. Differences were considered significant at $\mathrm{p}<0.05$.

\section{ACKNOWLEDGMENTS}

The valuable aid of Dr Enrique Busó in DNA methylation analysis is greatly acknowledged. We thank Dr B. Vogelstein for the gift of the D-Mut1 cell line. CIBERONC is an initiative of the Carlos III Health Institute.

\section{CONFLICTS OF INTEREST}

The authors declare that no conflicts of interests exists.

\section{GRANT SUPPORT}

This work was supported by a grant from Generalitat Valenciana (PROMETEO 2013-005 to A.C.). ALR-C was a fellow of the Grisolia (2012/034) program.

\section{REFERENCES}

1. Hancock JF. Ras proteins: different signals from different locations. Nat Rev Mol Cell Biol. 2003; 4:373-84.

2. Schubbert S, Shannon K, Bollag G. Hyperactive Ras in developmental disorders and cancer. Nat Rev Cancer. 2007; 7:295-308.

3. Hancock JF, Magee AI, Childs JE, Marshall CJ. All ras proteins are polyisoprenylated but only some are palmitoylated. Cell. 1989; 57:1167-77.

4. Laude AJ, Prior IA. Palmitoylation and localisation of RAS isoforms are modulated by the hypervariable linker domain. J Cell Sci. 2008; 121:421-27.

5. Bivona TG, Quatela SE, Bodemann BO, Ahearn IM, Soskis MJ, Mor A, Miura J, Wiener HH, Wright L, Saba SG, Yim D, Fein A, Pérez de Castro I, et al. PKC regulates a farnesylelectrostatic switch on K-Ras that promotes its association with Bcl-XL on mitochondria and induces apoptosis. Mol Cell. 2006; 21:481-93.

6. Weise K, Kapoor S, Werkmüller A, Möbitz S, Zimmermann G, Triola G, Waldmann H, Winter R. Dissociation of the K-Ras4B/PDE $\delta$ complex upon contact with lipid membranes: membrane delivery instead of extraction. J Am Chem Soc. 2012; 134:11503-10.

7. Schmick M, Vartak N, Papke B, Kovacevic M, Truxius DC, Rossmannek L, Bastiaens PI. KRas localizes to the plasma membrane by spatial cycles of solubilization, trapping and vesicular transport. Cell. 2014; 157:459-71. 
8. Dharmaiah S, Bindu L, Tran TH, Gillette WK, Frank PH, Ghirlando R, Nissley DV, Esposito D, McCormick F, Stephen AG, Simanshu DK. Structural basis of recognition of farnesylated and methylated KRAS $4 b$ by PDE $\delta$. Proc Natl Acad Sci USA. 2016; 113:E6766-75.

9. Plowman SJ, Berry RL, Bader SA, Luo F, Arends MJ, Harrison DJ, Hooper ML, Patek CE. K-ras 4A and 4B are co-expressed widely in human tissues, and their ratio is altered in sporadic colorectal cancer. J Exp Clin Cancer Res. 2006; 25:259-67.

10. King S, Bray S, Galbraith S, Christie L, Fleming S. Evidence for aldosterone-dependent growth of renal cell carcinoma. Int J Exp Pathol. 2014; 95:244-50.

11. Plowman SJ, Williamson DJ, O’Sullivan MJ, Doig J, Ritchie AM, Harrison DJ, Melton DW, Arends MJ, Hooper ML, Patek CE. While K-ras is essential for mouse development, expression of the K-ras 4A splice variant is dispensable. Mol Cell Biol. 2003; 23:9245-50.

12. Wang Y, You M, Wang Y. Alternative splicing of the K-ras gene in mouse tissues and cell lines. Exp Lung Res. 2001; 27:255-67.

13. Voice JK, Klemke RL, Le A, Jackson JH. Four human ras homologs differ in their abilities to activate Raf-1, induce transformation, and stimulate cell motility. J Biol Chem. 1999; 274:17164-70.

14. Plowman SJ, Arends MJ, Brownstein DG, Luo F, Devenney PS, Rose L, Ritchie AM, Berry RL, Harrison DJ, Hooper ML, Patek CE. The K-Ras 4A isoform promotes apoptosis but does not affect either lifespan or spontaneous tumor incidence in aging mice. Exp Cell Res. 2006; 312:16-26.

15. Patek CE, Arends MJ, Rose L, Luo F, Walker M, Devenney PS, Berry RL, Lawrence NJ, Ridgway RA, Sansom OJ, Hooper ML. The pro-apoptotic K-Ras 4A proto-oncoprotein does not affect tumorigenesis in the ApcMin/+ mouse small intestine. BMC Gastroenterol. 2008; 8:24.

16. Chung SI, Moon H, Ju HL, Kim DY, Cho KJ, Ribback S, Dombrowski F, Calvisi DF, Ro SW. Comparison of liver oncogenic potential among human RAS isoforms. Oncotarget. 2016; 7:7354-66. https://doi.org/10.18632/ oncotarget.6931.

17. Luo F, Ye H, Hamoudi R, Dong G, Zhang W, Patek CE, Poulogiannis G, Arends MJ. K-ras exon 4A has a tumour suppressor effect on carcinogen-induced murine colonic adenoma formation. J Pathol. 2010; 220:542-50.

18. To MD, Wong CE, Karnezis AN, Del Rosario R, Di Lauro $\mathrm{R}$, Balmain A. Kras regulatory elements and exon 4A determine mutation specificity in lung cancer. Nat Genet. 2008; 40:1240-44.

19. Abubaker J, Bavi P, Al-Haqawi W, Sultana M, Al-Harbi S, Al-Sanea N, Abduljabbar A, Ashari LH, Alhomoud S, Al-Dayel F, Uddin S, Al-Kuraya KS. Prognostic significance of alterations in KRAS isoforms KRAS-4A/4B and KRAS mutations in colorectal carcinoma. J Pathol. 2009; 219:435-45.
20. Kornblihtt AR, Schor IE, Alló M, Dujardin G, Petrillo E, Muñoz MJ. Alternative splicing: a pivotal step between eukaryotic transcription and translation. Nat Rev Mol Cell Biol. 2013; 14:153-65.

21. Naftelberg S, Schor IE, Ast G, Kornblihtt AR. Regulation of alternative splicing through coupling with transcription and chromatin structure. Annu Rev Biochem. 2015; 84:165-98.

22. Dassano A, Colombo F, Trincucci G, Frullanti E, Galvan A, Pettinicchio A, De Cecco L, Borrego A, Martinez Ibañez OC, Dragani TA, Manenti G. Mouse pulmonary adenoma susceptibility 1 locus is an expression QTL modulating Kras-4A. PLoS Genet. 2014; 10:e1004307.

23. Tsai FD, Lopes MS, Zhou M, Court H, Ponce O, Fiordalisi JJ, Gierut JJ, Cox AD, Haigis KM, Philips MR. K-Ras4A splice variant is widely expressed in cancer and uses a hybrid membrane-targeting motif. Proc Natl Acad Sci USA. 2015; 112:779-84.

24. Lev Maor G, Yearim A, Ast G. The alternative role of DNA methylation in splicing regulation. Trends Genet. 2015; 31:274-80.

25. Gelfman S, Ast G. When epigenetics meets alternative splicing: the roles of DNA methylation and GC architecture. Epigenomics. 2013; 5:351-53.

26. Hu Q, Kim EJ, Feng J, Grant GR, Heller EA. Histone posttranslational modifications predict specific alternative exon subtypes in mammalian brain. PLoS Comput Biol. 2017; 13:e1005602.

27. Michalak EM, Visvader JE. Dysregulation of histone methyltransferases in breast cancer - Opportunities for new targeted therapies? Mol Oncol. 2016; 10:1497-515.

28. Barbier J, Dutertre M, Bittencourt D, Sanchez G, Gratadou L, de la Grange P, Auboeuf D. Regulation of H-ras splice variant expression by cross talk between the p53 and nonsense-mediated mRNA decay pathways. Mol Cell Biol. 2007; 27:7315-33.

29. Jimeno-González S, Payán-Bravo L, Muñoz-Cabello AM, Guijo M, Gutierrez G, Prado F, Reyes JC. Defective histone supply causes changes in RNA polymerase II elongation rate and cotranscriptional pre-mRNA splicing. Proc Natl Acad Sci USA. 2015; 112:14840-45.

30. Shukla S, Kavak E, Gregory M, Imashimizu M, Shutinoski B, Kashlev M, Oberdoerffer P, Sandberg R, Oberdoerffer S. CTCF-promoted RNA polymerase II pausing links DNA methylation to splicing. Nature. 2011; 479:74-79.

31. Sacilotto N, Espert A, Castillo J, Franco L, López-Rodas G. Epigenetic transcriptional regulation of the growth arrest-specific gene 1 (Gas1) in hepatic cell proliferation at mononucleosomal resolution. PLoS One. 2011; 6:e23318.

32. Riffo-Campos ÁL, Castillo J, Tur G, González-Figueroa P, Georgieva EI, Rodríguez JL, López-Rodas G, Rodrigo MI, Franco L. Nucleosome-specific, time-dependent changes in histone modifications during activation of the 
early growth response 1 (Egr1) gene. J Biol Chem. 2015; 290:197-208.

33. Castillo J, López-Rodas G, Franco L. Histone posttranslational modifications and nucleosome organisation in transcriptional regulation: some open questions. Adv Exp Med Biol. 2017; 966:65-92.

34. Barash Y, Calarco JA, Gao W, Pan Q, Wang X, Shai O, Blencowe BJ, Frey BJ. Deciphering the splicing code. Nature. 2010; 465:53-59.

35. Spies N, Nielsen CB, Padgett RA, Burge CB. Biased chromatin signatures around polyadenylation sites and exons. Mol Cell. 2009; 36:245-54.

36. Luco RF, Allo M, Schor IE, Kornblihtt AR, Misteli T. Epigenetics in alternative pre-mRNA splicing. Cell. 2011; 144:16-26.

37. Sims RJ 3rd, Millhouse S, Chen CF, Lewis BA, ErdjumentBromage H, Tempst P, Manley JL, Reinberg D. Recognition of trimethylated histone $\mathrm{H} 3$ lysine 4 facilitates the recruitment of transcription postinitiation factors and premRNA splicing. Mol Cell. 2007; 28:665-76.

38. Gunderson FQ, Johnson TL. Acetylation by the transcriptional coactivator Gen5 plays a novel role in co-transcriptional spliceosome assembly. PLoS Genet. 2009; 5:e1000682.

39. Kelly AD, Issa JJ. The promise of epigenetic therapy: reprogramming the cancer epigenome. Curr Opin Genet Dev. 2017; 42:68-77.

40. Bonnal S, Vigevani L, Valcárcel J. The spliceosome as a target of novel antitumour drugs. Nat Rev Drug Discov. 2012; 11:847-59.
41. Roda D, Castillo J, Telechea-Fernández M, Gil A, LópezRodas G, Franco L, González-Rodríguez P, Roselló S, Pérez-Fidalgo JA, García-Trevijano ER, Cervantes A, Zaragozá R. EGF-induced acetylation of heterogeneous nuclear ribonucleoproteins is dependent on KRAS mutational status in colorectal cancer cells. PLoS One. 2015; 10:e0130543.

42. Riffo-Campos Á, Castillo J, Vallet-Sánchez A, Ayala G, Cervantes A, López-Rodas G, Franco L. In silico RNA-seq and experimental analyses reveal the differential expression and splicing of EPDR1 and ZNF518B genes in relation to KRAS mutations in colorectal cancer cells. Oncol Rep. 2016; 36:3627-3634.

43. Xi L, Fondufe-Mittendorf Y, Xia L, Flatow J, Widom J, Wang JP. Predicting nucleosome positioning using a duration Hidden Markov Model. BMC Bioinformatics. 2010; 11:346.

44. Coolen MW, Statham AL, Gardiner-Garden M, Clark SJ. Genomic profiling of $\mathrm{CpG}$ methylation and allelic specificity using quantitative high-throughput mass spectrometry: critical evaluation and improvements. Nucleic Acids Res. 2007; 35:e119.

45. Sandoval J, Rodríguez JL, Tur G, Serviddio G, Pereda J, Boukaba A, Sastre J, Torres L, Franco L, López-Rodas G. RNAPol-ChIP: a novel application of chromatin immunoprecipitation to the analysis of real-time gene transcription. Nucleic Acids Res. 2004; 32:e88.

46. Tur G, Georgieva EI, Gagete A, López-Rodas G, Rodríguez JL, Franco L. Factor binding and chromatin modification in the promoter of murine Egrl gene upon induction. Cell Mol Life Sci. 2010; 67:4065-77. 